\title{
Peritoneal dialysis versus hemodialysis in the management of sickle cell disease patients with end- stage renal disease: an observational study
}

\begin{abstract}
There is paucity of studies that discussed the role of peritoneal dialysis (PD) in managing end stage renal disease (ESRD) in sickle cell disease (SCD) patients. The present study compares the outcome of SCD-ESRD patients treated with hemodialysis (HD) or PD. Sixty incident ESRD patients were allotted to HD, (HD group, $\mathrm{n}=40$ ) or PD, (PD group, $\mathrm{n}=20$ ). Causes and severity of renal injury were assessed at the time of initiating dialysis. The primary outcome was hospital mortality at 5 years, and secondary outcomes were infection rates, incidents of vasoocclusive crisis (VOC) and acute chest syndrome (ACS), response to erythropoietic agents and improvement of cardiac function. No statistically significant differences were observed between groups in regard to patients' characteristics. The survival at 5 years was significantly better in the patients treated with PD when compared to HD $(75.0 \%$ vs. $57.5 \%, p=0.026)$. Infectious complications $(15 \%$ vs $35 \%, p<0.001)$, blood transfusion requirements $(p<0.001)$, VOC $(15 \%$ vs. $42.5 \%, p<0.001)$ and ACS $(10 \%$ vs $27.5 \%, p<0.001$ ) were significantly less in the PD group. Response to erythropoietic agents and improvement of left ventricular ejection fraction (LVEF) were significantly better in the PD group ( $p=0.022$ and $p<0.001$, respectively). This study suggests that there are better outcomes with PD compared to HD in the treatment of SCD-ESRD patients with differen dialysis modalities. Key Words: SCD, HD, PD, erythropoiesis, blood transfusion, VOC, ACS, sepsis, cardiac function, survival.
\end{abstract}

Keywords: end stage renal failure, peritoneal dialysis, sickle cell disease
Volume 9 Issue 2 - 202I

\author{
Abdullah Alhwiesh,' Ibrahiem Saeed \\ Abdul-Rahman,' Abdulla Al-Shehri, ${ }^{2}$ Amani \\ Alhwiesh,' Nadia Al-Audah,' Abdulla \\ Abdulrahman, ${ }^{4}$ Abdelgalil Moaz Mohammed,' \\ Waleed Al-Bakr,' Mohamad Sakr,' Nehad Al- \\ Audah, ' Ali Alharbi, ${ }^{3}$ Mousa Dujanah, ${ }^{3}$ Sami \\ Skhiri, ${ }^{3}$ Mohamed Nasr, ${ }^{3}$ Ismail Ibrahim, ${ }^{3}$ \\ Tajalkhatem Nourboja ${ }^{3}$ \\ 'Nephrology Division, Department of Internal Medicine, King \\ Fahd Hospital of the University, Imam Abdulrahman Bin Faisal \\ University, Saudi Arabia \\ ${ }^{2}$ Cardiology Division, Department of Internal Medicine, King \\ Fahd Hospital of the University, Imam Abdulrahman Bin Faisal \\ University, Saudi Arabia \\ ${ }^{3}$ Diaverum, Eastern Province \\ ${ }^{4}$ Department of Electrical Engineering, Queen's University, \\ Toronto, Kingston, Canada
}

Correspondence: Abdullah K.Al-Hwiesh, M.D, Professor and Consultant of Nephrology, Imam Abdulrahman Bin Faisal University, King Fahd Hospital of the University, Al-Khobar, 1952, Saudi Arabia, Email ahwiesh@iau.edu.sa

Received: July 20, 2021 | Published: August 19, 2021

\section{Introduction}

Patients with sickle cell disease (SCD) comprised only 410 of the 442,017 patients with end-stage renal disease (ESRD) who started hemodialysis in the US Centers for Medicare and Medicaid systems. The relatively small size of the SCD-ESRD population has limited the development of optimal management strategies. Hemodialysis (HD) is reportedly the leading form of renal replacement therapy for SCDESRD patients, ${ }^{2}$ but other dialysis modalities for these patients also include peritoneal dialysis (PD). Both HD and PD may confer their own theoretical advantages. Hemodialysis may be used for urgent or instant need for standard and exchange blood transfusions. In contrast, $\mathrm{PD}$ and its inherent slow rate of ultrafiltration may minimize any acute rise in hematocrit and thus lower the risk of vaso-occlusive crisis. ${ }^{3}$

Of interest, only $6.8 \%$ of SCD patients began dialysis with a functioning arteriovenous fistula, ${ }^{1}$ which rendered them plagued with the complications of repeated central venous catheterization. Mortality in SCD-HD patients is approximately $26 \%$ during the first year of therapy for ESRD, which is nearly threefold higher than in ESRD patients without SCD. ${ }^{1}$ However, SCD patients who received pre-dialysis nephrology care had a lower death rate than those who did not receive such care. ${ }^{1}$

The management of anemia with erythropoietic agents is vital for the global treatment of sickle cell disease-dialysis patients. Anemia in dialysis patients with SCD is a unique challenge. There are reports suggesting that anemia control with erythropoietic agents is obtained more easily in PD patients than in HD patients. This was demonstrated in a study by Coronel $\mathrm{F}$, et al. who showed that with the same administration route, PD patients showed a reduced erythropoietic agents' requirement, and less frequent administration than HD patients. ${ }^{4}$ In contrast, a meta-analysis was recently published aiming at evaluating the effect of PD and HD on renal anemia showed that both of the two therapeutic strategies had a similar effect on anemia in renal disease patients. 5

Several studies demonstrated high prevalence of vasoocclusive crisis during or following hemodialysis sessions. ${ }^{3,6,7}$ One possible explanation for this finding is that salt and water retention before hemodialysis initiation predispose patients to a state of relative hypoxia, a well-known trigger factor for VOC. ${ }^{3}$ Conversely, after dialysis initiation, excessive fluid load variation, with a gradual decrease in body dry weight, might promote VOC occurrence. ${ }^{8}$ Whether or not the same applies to PD treatment is not yet clear.

The 5-year cumulative incidence of death observed in sickle cellHD patients is relatively high when compared with dialysis non-sickle disease patients. ${ }^{1,8}$ Theoretically, PD might be considered as a more practical dialysis modality. The slow, more continuous ultrafiltration that occurs during PD eliminates the sharp rise in hematocrit seen during conventional thrice weekly HD treatments and the potentially harmful conditions of an extracorporeal circuit and hence might decrease dialysis morbid complications. 
As for the present time, there are no data to assess the relative risk and benefits of HD vs. PD in SCD patients; this study aims at comparing the two dialysis modalities (HD \& PD) in terms of complications, need for erythropoietic agents, cardiac function as well as survival.

\section{Patients and methods}

This is a prospective observational study that was conducted in two big dialysis centers; King Fahd Hospital of the University HD \& PD centers and Diaverum hemodialysis center in the Eastern Province, Saudi Arabia. The study aimed at comparing the outcomes of two modalities of renal replacement therapy (HD vs. PD) in sickle cell disease-ESRD patients after 5-year therapy. The total number of SCD-ESRD patients was 89 at initiation of the study, only sixty patients; 37 males and 23 females with SCD-ESRD patients were enrolled in our study and followed up between January 2015 and February 2020. Twenty-nine patients were excluded because of dialysis access malfunction, ${ }^{4}$ severe sepsis necessitating transfer to ICU, ${ }^{2}$ shift from PD to HD or vice versa during the study period, severe aplastic complication necessitating bone marrow transplant in a different facility, ${ }^{2}$ Patients with acute kidney injury requiring less than 3 months renal replacement therapy, ${ }^{5}$ SCD-ESRD patients who did not complete their ECHO study, ${ }^{6}$ and patients' refusal to participate. ${ }^{2}$ In addition, 3 patients left the study early as they were subjected to kidney transplantation shortly after the study initiation, so, only 60 patients were included in the statistical analysis; 40 incident HD and 20 incident PD patients. Demographic data, blood pressure and other clinical data were recorded during each routine physical examination. Clinical variables including age at diagnosis of SCD, the frequency of vaso-occlusive crises, number of hospitalizations per year, infection rates, dose of erythropoietic agent needed and transfusion therapy were collected. Laboratory data including hematological profile, renal function test was also obtained. The glomerular filtration rate was estimated (eGFR) using the 'modification of diet in renal disease' (MDRD) formula. ${ }^{9}$ The outcomes on incident HD-SCD patient were compared to an age matched group of SCD patients on PD. The data of the patients were obtained from the routine monthly dialysis recordings. Unscheduled haemoglobin measurements recorded in the emergency room during painful episodes and control haemoglobin levels measured after transfusions were recorded but not included in the calculations. The means of the haemoglobin and ferritin levels, transferrin saturation indices, blood transfusion requirements and hospitalizations were obtained before and after treatment with darbepoetin alfa over the study period. The results of pre- and postdarbepoetin treatment were compared using the paired $t$-test. The study was conducted in accordance with the Declaration of Helsinki and local laws and regulations as it was approved by the KFHU Ethical Committee. All patients were 18-year-old or more and an informed written consent was obtained from every patient. Follow-up for each patient began on the day of the first dialysis treatment and patients who survived were censored (their follow-up ended) at the completion of the study period.

\section{Data collected for SCD patients}

Excluding the 6 patients who did not have a complete ECHO study, the demographic, clinical and laboratory data were assessed for each patient at the time of the initiation of dialysis, at 12-month period and at the end of study then after.

\section{Dialysis technique}

HD was carried out three-times per week for four to four and half hours as needed. Dialysis was initiated through A-V fistula if suitable otherwise through central venous catheter (CVC). HD was performed with registered well-trained staff nurses and complete aseptic recommendations were followed as with our dialysis protocol. dialyzer (high flux) being $140 \mathrm{H}, 170 \mathrm{H}$ or $210 \mathrm{H}$ (Gambro Dialysatoren, Holger-Craford-Strasse, Hechingen, Germany) depending on the patient's surface area. Acid concentrate (ADAMCO, limited, SA) and bicarbonate (Bicart, Gambro, Lundia $\mathrm{AB}$, Sweden) were used in all patients.

All PD catheters were inserted following Al-Hwiesh technique (10) by one well-trained nephrology team. All Patients were on automated peritoneal dialysis (APD) and their dialytic prescription consisted of $1.36 \%$ and $2.27 \%$ glucose-based solutions Dianeal $\AA$ over 9-10 hours night dwell and 7.5\% icodextrin (Extraneal ${ }^{\circledR}$, Baxter Castlebar, Ireland) 2 liters as the last fill for the day dwell if needed. Total daily PD volume ranged between 10-12 liters with a fill volume ranging between 2.0-2.5 liters/cycle.

\section{Statistical analysis}

All the relevant data were obtained from the patient hemodialysis and radiology records and the hospital blood result computer database. Statistical analysis was performed using SPSS software (SPSS Inc., Chicago, IL; (version 20). Results are shown as average (minimum \& maximum) or mean $\pm \mathrm{SD}$. The student t-test was used for continuous variables and chi-square for categorical variable for comparisons of means and proportions amongst subgroups. $P$ values were calculated using spreadsheets/statistical software. These calculations are based on the assumed or known probability distribution of the specific statistic being tested. The 5-year survival of the two groups of patients were compared using a spread Excel sheet.

\section{Results}

For the 40 SCD-HD patients and the 20 SCD-PD patients who completed the study, the mean $\pm \mathrm{SD}$ serum creatinine at dialysis initiation and at the end of study were $8.8 \pm 0.77$ and $6.4 \pm 0.81$ vs. $8.4 \pm 0.83$ and $4.1 \pm 0.44 \mathrm{mg} / \mathrm{dl}$ respectively $(p=0.002)$. The estimated glomerular filtration rate was $13.6 \pm 3.7$ and $13.8 \pm 3.3 \mathrm{ml} / \mathrm{min}$ per $1.73 \mathrm{~m}^{2}(p=0.15)$. Following the diagnosis of renal disease, renin-angiotensin system (RAS) inhibitor treatment was initiated in $19(47.5 \%)$ HD-SCD patients and in $10(50.0 \%)$ PD-SCD patients $(p=0.133)$. Demographic and biological data at the start of renal replacement therapy are summarized in Table 1.

At the start of dialysis, a functioning AVF was reported present among $8(20 \%)$ of patients with HD-SCD, immature AVF in 11 $(27.5 \%)$. The majority of individuals with HD-SCD (80\%) started dialysis with a central venous catheter, and were more likely to have a catheter for the first hemodialysis.

All hemodialysis patients were started on darbepoetin alfa $0.75 \mathrm{ug} /$ $\mathrm{kg} /$ week sc. when hemoglobin levels were equal to or less than $7 \mathrm{~g} /$ dl. Despite this therapy, haemoglobin concentrations fell to 5.5-6.5 g/ $\mathrm{dL}$ in 2 months in $13(32.5 \%)$ HD patients. Therefore, the darbepoetin alfa dosage was increased to the upper end of the dosage range allowed for ESRD patients, according to guidelines. However, there was no satisfactory response to the erythropoietic agents as Hgb levels kept at the same level of $\leq 7 \mathrm{gm} / \mathrm{dl}$. In the remaining $27(67.5 \%)$ haemoglobin levels were maintained at an average of $8.4 \mathrm{gm} / \mathrm{dl}$ with the maximum allowed dose of darbepoetin alfa. Of all the SCD-PD patients, $3(15 \%)$ patients were recorded as being resistant to the maximum allowed darbepoetin dose with an average $\mathrm{Hgb}$ level $\leq 7 \mathrm{~g} / \mathrm{dl}$ throughout the study period $(p=0.022)$. Eighteen ( $45 \%)$ HD patients and one $(5 \%) \mathrm{PD}$ patients needed hydroxyurea (with an adjusted ESRD dose $(500 \mathrm{mg} /$ day) to prevent repeated VOC during the study period $(p<0.001)$. No patient was receiving iron therapy. 
The proportion of patients with at least one episode of vasoocclusive crisis (VOC) requiring hospitalization was higher in the first year after hemodialysis initiation than in the SCD-PD patients [17 $(42.5 \%)$ vs. $3(15 \%) ; p<0 \cdot 001]$. Similarly, acute chest syndrome requiring intensive care unit admission was reported in $11(27.5 \%)$ of HD and $2(5 \%)$ in PD patients $(p<0.001)$ (Table 2), and although more patients with SCD were admitted because of acute coronary syndrome in the $1^{\text {st }} \mathrm{HD}$ year, yet they were as likely to have it as the other patients on PD (12.5\% vs. $10.0 \%,(p=0.233)$.

Table I Patients' characteristics at dialysis initiation

\begin{tabular}{|c|c|c|}
\hline Characteristics & SCD-HD patients $(n=40) n \%$ & SCD-PD $(n=20) n \%$ \\
\hline Age (mean + SD) & $38.21+7.59$ & $37.66+8.72$ \\
\hline \multicolumn{3}{|l|}{ Gender } \\
\hline Female & 1640.0 & 735.0 \\
\hline Male & 2460.0 & 1365.0 \\
\hline Predialysis care BMI $\left(\mathrm{kg} / \mathrm{m}^{2}\right)$ & 3177.5 & 1470.0 \\
\hline$<25$ & 2767.5 & 1365.0 \\
\hline$>25$ & 1332.5 & 735.0 \\
\hline DM & 37.5 & 210.0 \\
\hline $\mathrm{IHD}$ & 512.5 & 315.0 \\
\hline $\mathrm{CHF}$ & 512.5 & 210.0 \\
\hline PVD & 717.5 & 315.0 \\
\hline HTN & 820.0 & 525.0 \\
\hline $\mathrm{Hgb}<7 \mathrm{gm} / \mathrm{dl}$ & 2767.5 & 1365.0 \\
\hline Serum Cr. $(\mathrm{mg} / \mathrm{dl})$ mean $+\mathrm{SD}$ & $8.8 \pm 0.77$ & $8.4+0.83$ \\
\hline Initial GFR $\left(\mathrm{ml} / \mathrm{min} / \mathrm{l} \cdot 73 \mathrm{~m}^{2}\right)$ mean + SD & $13 \cdot 6 \pm 3 \cdot 7$ & $13.8+3.3$ \\
\hline Treatment with ARB & 1947.5 & 1050.0 \\
\hline
\end{tabular}

SCD-HD, sickle cell disease hemodialysis group; BMI, body mass index; DM, diabetes mellitus; IHD, ischemic heart disease; CHF, congestive heart failure; PVD, peripheral vascular disease; HTN, hypertension; Hgb, hemoglobin; $\mathrm{Cr}$, creatinine; GFR, glomerular filtration rate; ARB, angiotensin receptor blocker

Table 2 Outcomes

\begin{tabular}{|c|c|c|c|}
\hline Measure & SCD-HD n \% & SCD-PD n \% & $\mathbf{p}$ \\
\hline VOC & 1742.5 & 315 & $<0.001$ \\
\hline Hemolytic crisis & 37.5 & 15 & 0.268 \\
\hline Aplastic crisis & 37.5 & 00 & 0.021 \\
\hline ACS & | 27.5 & 210 & $<0.001$ \\
\hline \multicolumn{4}{|l|}{ Need for blood transfusion } \\
\hline - At the beginning & Minimum I-2 U/2-3 m & Minimum I-2U/8 m & $<0.001$ \\
\hline - At the end & Maximum I-2 U/I-2 m & Maximum I-2U/ 4-6 m & \\
\hline dialysis catheter mechanical complication & II 27.5 & 210.0 & 0.002 \\
\hline Dialysis axis infection & 922.5 & 210.0 & 0.013 \\
\hline Septicemia & 512.5 & 15.0 & 0.017 \\
\hline Thromboembolic events & 615.0 & 15.0 & $<0.01$ \\
\hline Acute coronary syndrome & $5 \mid 2.5$ & 210 & 0.371 \\
\hline - Initially & $36.4 \pm 7.7$ & $36.6 \pm 5.4$ & \\
\hline - At I2-month & $37.7 \pm 6.3$ & $40.4 \pm 4.9$ & $<0.001$ \\
\hline - At 5 years & $37.1+5.3$ & $46.3+5.0$ & \\
\hline
\end{tabular}




\begin{tabular}{llll} 
Table Continued... & SCD-HD n & SCD-PD n & P \\
\hline Measure & & & \\
\hline ECHO & 2357.5 & 630.0 & 0.006 \\
- RVD & 1947.5 & 420.0 & $<0.001$ \\
- LAD & 1947.5 & 315.0 & $<0.001$ \\
- Increased LVWT & $5.5(3-8)$ & $2.5(2-3)$ & 0.04 \\
Number of Hospitalization-year (average) & 2357.5 & 1575.0 & 0.026 \\
5-year survival & &
\end{tabular}

SCD, sickle cell disease; HD, hemodialysis; PD, peritoneal dialysis;VOC, vasoocclusive crisis; ACS, acute chest syndrome; CVC, central venous catheter; LVEF, left ventricular ejection fraction; RVD, right ventricular dilatation; LAD, left atrial dilatation; LVWT, left ventricular wall thickness

In the HD group, there was no significant difference between the means of the haemoglobin $(6.8 \pm 0.4$ vs $7.7 \pm 0.3 \mathrm{~g} / \mathrm{dL})$, ferritin levels $(1022 \pm 214$ vs $1051 \pm 218 \mathrm{ng} / \mathrm{mL})$, transferrin saturation indices $(74.6 \pm 16.4$ vs $77.1 \pm 18.2 \%)$, and blood transfusion needs and rates (minimum 1-2 U/2-3 months, maximum 1-2 U/1-2 months) at the beginning and at the end of the study. In the PD group of SCD patients, there were remarkable differences of hemoglobin levels ( $7.1 \pm 0.8$ vs. $9.2 \pm 1.2 \mathrm{~g} / \mathrm{dl})$, ferritin levels (1105 $\pm 194 \mathrm{vs} .688 \pm 156 \mathrm{ng} /$
$\mathrm{mL})$, transferrin saturation $(75.5 \pm 14.8$ vs. $62.2 \pm 11.8 \%)$ and blood transfusion needs and rates (minimum 1-2 U/8 months, maximum 1-2 U/4-6 months), $p<0.001$. Six (15.0\%) HD patients and one $(5.0 \%)$ of the PD patients had newly developed thromboembolic event during the study period $(p<0.01)$. Number of hospitalizations in the HD group was significantly more than in the PD group (5.5 times per year-min. 3, max. 8 vs 2.5 times per year-min. 2, max. 3) (Table 2 \& Figure 1).

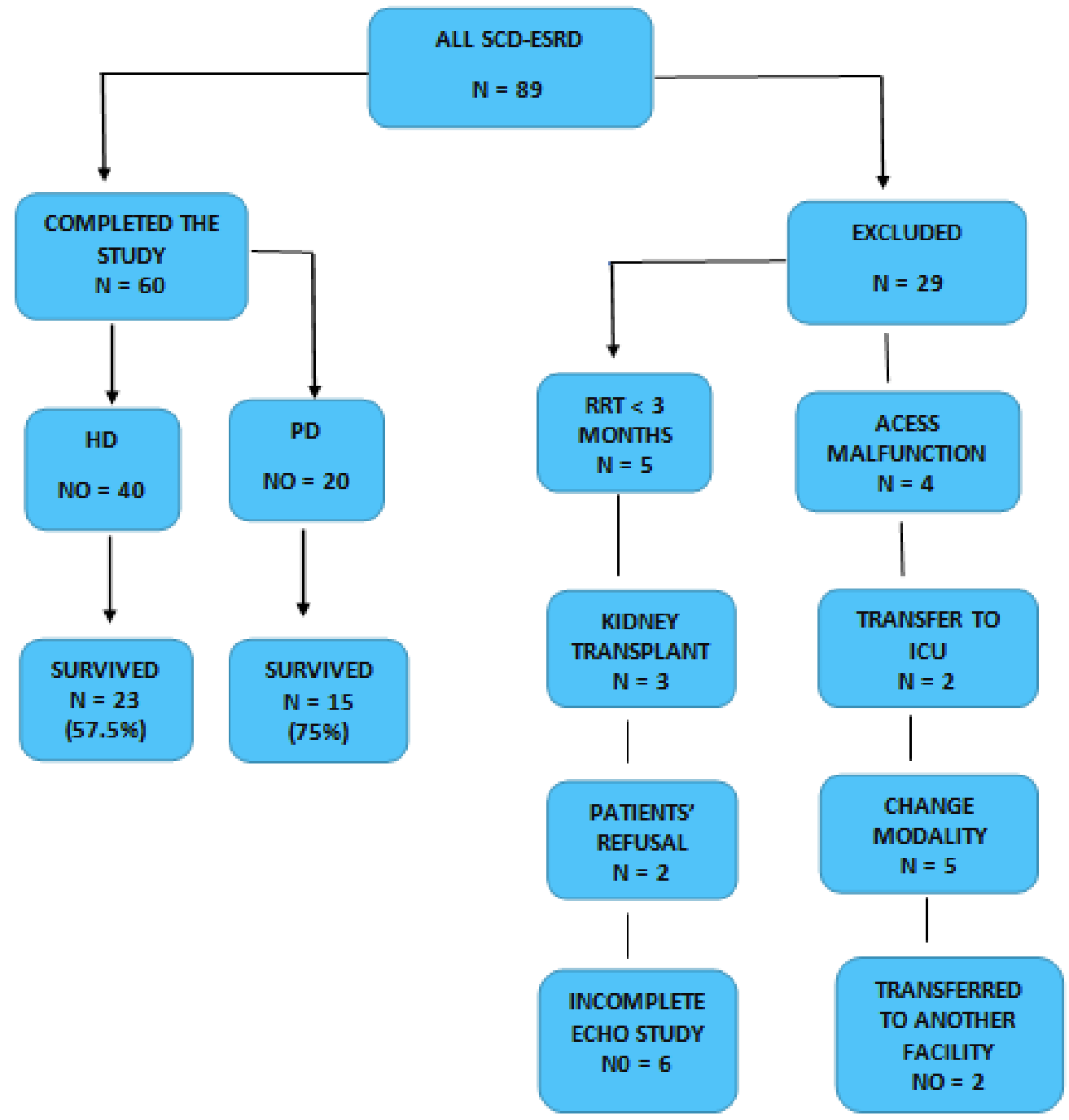

Figure I Diagram illustrating the enrolled and excluded SCD-ESRD patients with HD \& PD. 
All enrolled Patients of the two groups underwent transthoracic echocardiography at HD and PD initiation (Table-2), at 12 months and at the end of study. Left ventricle ejection fraction (LVEF) increased significantly in the PD group when compared with SCD patients on HD between these time-points; for HD mean $36 \cdot 4 \% \pm 7.7,37.7 \% \pm 6.3$ and $37.1 \% \pm 5.3$ at dialysis initiation, at 12 months and at termination of the study, respectively $(p=0 \cdot 387)$. In the PD patients LVEF increased from $36.6 \% \pm 5.4$ at dialysis initiation to $40.4 \% \pm 4.9$ at 12 months and $46.3 \% \pm 5.0$ at the end of study $(p<0.001)$ (Table $2 \&$ Figure 1$)$. After a median (IQR) follow-up of 38 months (36-57), acute pulmonary edema requiring hospitalization was the most frequent cardiovascular complication observed in SCD-HD patients during the study period $(9,22.5 \%)$. This complication was reported in $2(10 \%)$ of those who were treated with PD modality $(p=0.024)$.

Compared to $\mathrm{PD}$; infection rates related to dialysis procedure were significantly higher in SCD-HD patients ( $35 \%$ vs. $15 \%, p<0.001)$.
In the HD patients CVC infections accounted for 7 (17.5\%), AVF infection $2(5 \%)$, septicemia requiring admission $5(12.5 \%)$ patients while peritonitis was reported in $2(10 \%)$ and septicemia requiring admission in 1 (5\%) of PD patients during the study period (Table 2). At the end of the study mechanical complications related to dialysis access was reported in $11(27.5 \%)$ and $2(10 \%)$ in the HD and PD groups, respectively $(p<0.002)$ (Table 2$)$.

The 5- year survival in patients receiving PD was significantly higher in the SCD-PD group than in the HD group $16(75.0 \%)$ vs. 25 $(57.5 \%)$ patients, respectively $(p=0.026$ ) (Figure 2$)$. Causes of death were septic shock in 6 , acute chest syndrome in 6 , acute pulmonary embolism in 4 and cerebral hemorrhage in $1 \mathrm{HD}$ patients, while death in the PD patients was due to acute chest syndrome in 2 , acute pulmonary embolism in 2 and acute extensive myocardial infarction in 1 patient.

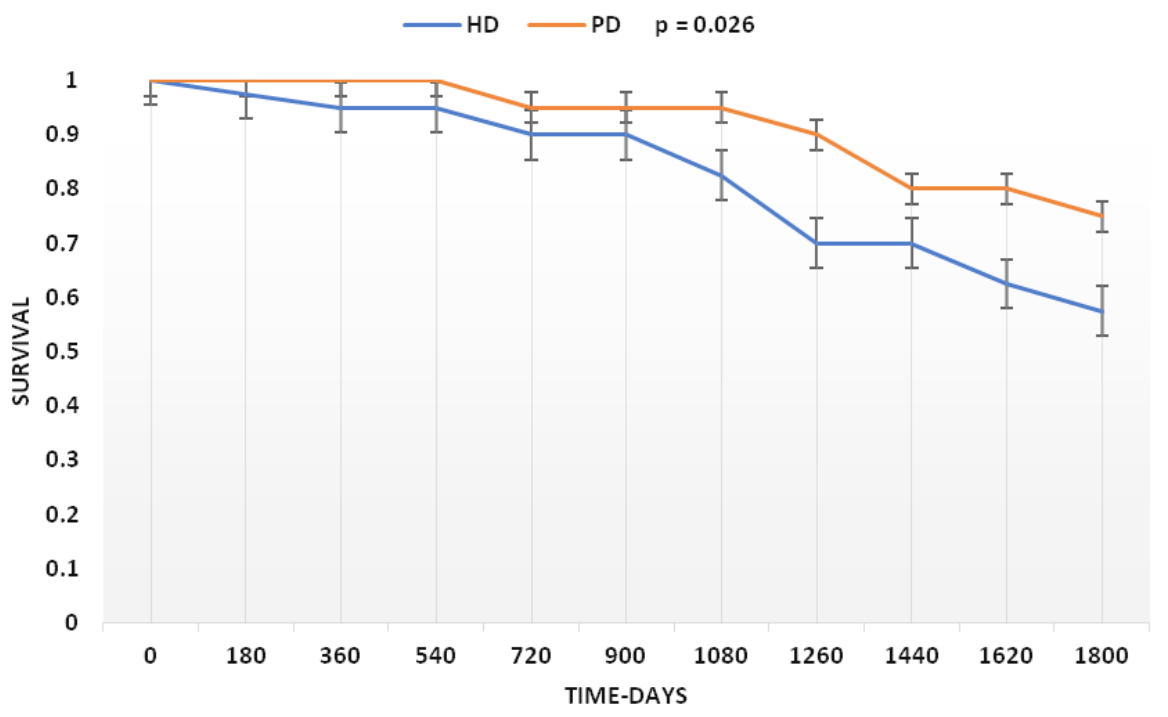

Figure 2 Survival (up to I,800 days) of SCD-HD vs. SCD-PD patients with ESRD.

\section{Discussion}

End-stage renal disease (ESRD) due to sickle cell anemia (SCA) occurs in $<1 \%$ of patients receiving renal replacement treatment. ${ }^{11}$ For this reason, data about this group of patients are very limited and controversial. Why does ESRD carry such a dismal prognosis in SCD patients? There are several possible explanations. Firstly, starting dialysis may have been unduly delayed, allowing the development of severe complications of chronic kidney disease. Secondly, undernutrition and underdialysis are well known to confer increased morbidity and mortality in sickle cell patients receiving dialysis. ${ }^{12,13}$ The third, and probably most important possibility, is that patients with sickle cell disease also have more extensive damage to other organs, particularly cardiac damage, than patients without SCD. ${ }^{14}$ The pathogenesis of cardiac disease may include chronic anemia, sickling in the coronary microcirculation, iron overload, and sickling in the pulmonary circulation..$^{15-17}$ The timing of initiation of dialysis treatment should depend as much on clinical features such as diuretic-resistant fluid retention, anorexia and nausea/vomiting rather than waiting for the serum blood urea nitrogen or creatine to reach pre-defined values..$^{18}$ Once dialysis has been initiated in SCD patients, careful attention should be paid to avoidance of fluid overload, which may contribute to cardiac damage, toxins' removal and adequate nutrition and treatment of anemia. Attention should be paid to avoiding iron overload especially in multiply transfused patients. ${ }^{16,19}$

For several decades, PD has been considered a second-class treatment for patients who develop ESRD and it has been used less frequently in the developed world. ${ }^{20}$ This might be because of lack of PD experience, or knowledge by physicians and nephrologists working with such group of patients, the limitations attributed to PD, and/or a lack of appropriate resources.

This study was designed to compare the use of PD as a form of RRT with HD for the treatment of SCD-ESRD patients, focusing on the impact of the two therapies on mortality and outcomes in this group of hemodynamically unstable patients. In our study, the infectious complications were less common with PD when compared with HD. Dialysis axis manipulations throughout HD, in addition to the presumptive value of PD in inflammatory cytokines removal might be reasonable explanations. The study conducted by Dittrich et al. ${ }^{21}$ illustrated the importance of $\mathrm{PD}$ in removing the proinflammatory cytokines, particularly, interleukins that could directly relate to the phenomena of capillary leak syndrome, infections and organ dysfunction. In addition, it has been found that substances that may induce and/or potentiate inflammatory responses are effectively 
removed mainly by PD but not by dialyzer membranes such as those used in HD..$^{22-24}$ There is, however, an argument about removal of the pro-inflammatory cytokines being the reason for sepsis or a factor that modifies the sepsis phenotype. ${ }^{23}$ Removal of proinflammatory cytokines by PD may not be the only explanation for the significantly lower incidence of sepsis in our PD population when compared with the HD group. Repeated central vein catheterization by different operators with all its drawbacks, handling of the catheter site by different nurse-staff teams and in some cases the need to change the whole set more than one time because of clotting and other problems may also be responsible. CVC devices are plagued by their propensity for infection, thrombosis, inadequate blood flow, damage to large central veins, overall cost and increased mortality risk which make their use problematic. CVC-related bacteremia averages between 3.4 and 5.5 incidences per 1000 catheter days with the resultant potential for the development of complications including sepsis, endocarditis and death..$^{25}$

In our study, there were significant differences between HD \& PD therapy in the number of patients with VOC and ACS episodes with an increased prevalence among sickle-HD patients. One possible explanation for this finding is that salt and water retention before HD initiation predispose patients to a state of relative hypoxia, a wellknown trigger factor for VOC. Following HD initiation, excessive fluid load variation, with a rapid decrease in body dry weight, might promote VOC occurrence. The slow, more continuous ultrafiltration that occurs during PD eliminates the sharp rise in hematocrit seen during conventional thrice weekly HD treatments and the potentially prosickling conditions of an extracorporeal circuit. ${ }^{3}$

Cardiac complications are a common feature of SCD and are felt to be an important cause of the morbidity and mortality associated with this disease. ${ }^{26-29}$ In our study, regular HD during the study period was associated with non-significant changes in estimated systolic LVEF in SCD patients. Chronic uncorrected anemia combined with iron overload in SCD-HD may have resulted in the cardiac chamber dilation and a compensatory increase in left ventricular mass shown in our study. In previous studies, this has been accompanied by left ventricular diastolic dysfunction which has also been a strong independent predictor of mortality in patients with SCD ${ }^{30}$ Compared with $\mathrm{HD}$, the remarkable response to erythropoietic therapy and the less need for blood transfusion with decreased chance for iron overload and above all the beneficial effect of PD in lowering the pulmonary artery pressure may all be reasonable explanations for the favorable cardiac performance with PD in our study. Nielsen et al. ${ }^{8}$ and McClellan et al. ${ }^{1}$ reported higher mortality rates in patients with ESRD-SCD when compared with non-SCD. Additionally, we found that patients with SCD-HD had higher mortality compared with PD-SCD patients despite their matching of age, blood pressure and comorbid conditions, such as DM, that are associated with lower survival in the general dialysis population.

There are two main drawbacks of our study; first, the sample size is relatively small and second, there are no tissue biopsy to confirm the presence of iron overload. However, as far as we know, this is the first study that compared the outcome of HD vs. PD-SCD patients with ESRD. We think that larger number of patients are needed to confirm our data.

\section{Conclusion}

This study suggests that there are better outcomes with PD compared to HD in the treatment of SCD-ESRD patients with different dialysis modalities.

\section{Declarations}

\section{Ethics approval and consent to participate and to publish}

After obtaining study-related approvals from the Ethics committee of King Fahd Hospital of the University, written informed consents to participate in and to publish the study was also obtained from all patients or their legal guardians.

Study protocols conformed to the ethical principles of medical research involving human subjects based on the Helsinki Declaration.

\section{Availability of data and materials}

All data and materials are presented in details within the manuscript.

\section{Declaration of conflicting (competing) interests}

The author(s) declared no potential conflicts of interest with respect to the research, authorship, and/or publication of this article.

\section{Funding}

The author(s) received no financial support for the research, authorship, and/or publication of this article.

\section{Acknowledgments}

The authors would like to express their sincere regards to all staff of hemodialysis and peritoneal dialysis unit and to extend their gratitude to the staff of the cardiology unit at King Fahd Hospital of the University, Saudi Arabia.

\section{References}

1. McClellan AC, Luthi JC, Lynch JR, et al. High one-year mortality in adults with sickle cell disease and end-stage renal disease. Br J Haematol. 2012;159(3):360-367.

2. Nachman P, Clark W, Derebail V. Nephrology Self-Assessment Program NephSAP. NephSAP. 2014;13:39-40.

3. Boyle SM, Jacobs B, Sayani FA, et al. Management of the Dialysis Patient with Sickle Cell Disease. Semin Dial. 2016;29(1):62-70.

4. Coronel F, Herrero JA, Montenegro J, et al. Erythropoietin requirements: a comparative multicenter study between peritoneal dialysis and hemodialysis. J Nephrol. 2003;16(5):697-702.

5. Wang WN, Zhang WL, Sun T, et al. Effect of peritoneal dialysis versus hemodialysis on renal anemia in renal in end-stage disease patients: a meta-analysis. Ren Fail. 2017;39(1):59-66.

6. Tsubakihara Y, Nishi S, Akiba T, et al. 2008 Japanese Society for Dialysis Therapy: Guidelines for renal anemia in chronic kidney disease. Therp Apher Dial. 2010;14(3):240-275.

7. Alkhunaizi AM, Al-Khatti AA, Al-Mueilo SH, et al. End-stage renal disease in patients with sickle cell disease. Saudi J Kidney Dis Transpl. 2017;28(4):751-757.

8. Nielsen L, Canou -Poitrine F, Jais JP, et al. Morbidity and mortality of sickle cell disease patients starting intermittent hemodialysis: a comparative cohort study with non- Sickle dialysis patients. Brit $J$ Hematol. 2016;174:148-152.

9. Levey AS, Bosch J, Lewis J, et al. A more accurate method to estimate glomerular filtration rate from serum creatinine: a new prediction equation. Modification of Diet in Renal disease study group. Annal Int Med. 1999;130:461-470. 
10. Al-Hwiesh AK. Percutaneous Peritoneal dialysis catheter insertion by a nephrologist: a new, simple, and safe technique. Perit Dial Int. 2014;34:204-211.

11. Zumrutdal A. Response of patients with sickle cell anemia and end-stage renal disease to erythropoietin treatment. NDT Plus. 2010;3(3):328-330.

12. Degoulet P, Legrain M, Reach I, et al. Mortality risk factors in patients treated by chronic hemodialysis. Nephron. 1982;31:103-110.

13. Hull AR, Parker TF. Proceedings from the morbidity, mortality and prescription of dialysis symposium, Dallas, TX, September 15 to 17 , 1989. Am J Kidney Dis. 1990;15:375-383.

14. Tomson CRV. End stage renal disease in sickle cell disease: future directions. Post Grad Med J. 1992;68:775-778.

15. Sundd P, Gladwin MT, Novelli EM. Pathophysiology of sickle cell disease. Annu Rev Pathol. 2019;14:263-292.

16. Liem RI, Lanzkron S, Coates TD, et al. American Society of Hematology 2019 Guidelines for Sickle Cell Disease: Cardiopulmonary and Kidney Disease. Blood Adv. 2019;3(23):3867-397.

17. Bakeer N, James J, Roy S, et al. Sickle cell anemia mice devrlop a unique cardiomyopathy with restrictive physiology. Proc Natl Acad Sci U S A. 2016;113(35):E5182-E5191.

18. Roy NBA, Fortin PM, Bull KR. Cochrane Cystic Fibrosis and Genetic Disorders Group. Cochrane Database Syst Rev. 2017;2017(7):CD012380.

19. Rostoker G, Vaziri ND, Fishbane S. Iatrogenic iron overload in dialysis patients at the beginning of the $21^{\text {st }}$ century. Drugs. 2016;76:741-57.

20. Mehrotra R, Devuyst O, Davis SJ, et al. The current state of peritoneal dialysis. J Am Soc Nephrol. 2016;27(11):3238-3252.
21. Dittrich S, Aktuerk D, Seitz S et al. Effects of ultrafiltration and peritoneal dialysis on proinflammatory cytokines during cardiopulmonary bypass surgery in newborns and infants. Eur J Cardiothorac Surg. 2004;25:935940.

22. Combe C, Pourtein M, De Precigout V, et al. Granulocyte activation and adhesion molecules during hemodialysis with cuprophane and a high-flux biocompatible membrane. Am J Kidney Dis. 1994;24:437-442.

23. Varela MP, Kimmel PL, Phillips TM, et al. Biocompatibility of hemodialysis membranes: interrelations between plasma complement and cytokine levels. Blood Purif. 2001;19:370-379.

24. Ronco C, Dell' Aquilla R, Rodighiero MP. Peritoneal Dialysis: A Clinical Update. Contrib Nephrol. Basel: Karger, 2006.

25. Nasser GM, Ayus JC. Infectious complications of the hemodialysis access. Kidney Int. 2001;60:1-13.

26. Adedeji MO, Cespedes J, Allen K, et al. Pulmonary thrombotic arteriopathy in patients with sickle cell disease. Arch Pathol Lab Med. 2001;125(11):1436- 41

27. Varat MA, Adolph RJ, Fowler NO. Cardiovascular effects of anemia. Am Heart J. 1972;83(3):415-426.

28. Rees DC, Williams TN, Gladwin MT. Sickle-cell disease. Lancet 2010;376:2018-2031

29. Powars DR, Elliott-Mills DD, Chan L, et al. Chronic renal failure in sickle cell disease: risk factors, clinical course and mortality. Ann Int Med. 1991;115(8):614-620.

30. Collins AJ, Foley RN, Herzog C, et al. United States Renal Data System 2008. Am J Kidney Dis. 2009;53(suppl 1):S1-374. 